\title{
Dispersive Quantization
}

\author{
Peter J. Olver ${ }^{\dagger}$ \\ School of Mathematics \\ University of Minnesota \\ Minneapolis, MN 55455 \\ olver@umn.edu \\ http: //www. math.umn. edu/ olver
}

\begin{abstract}
The evolution, through linear dispersion, of piecewise constant periodic initial data leads to surprising quantized structures at rational times, and fractal, nondifferentiable profiles at irrational times. Similar phenomena have been observed in optics and quantum mechanics, and lead to intriguing connections with exponential sums arising in number theory. Ramifications of these observations for numerics and nonlinear dispersion are proposed as open problems.
\end{abstract}

\section{Introduction.}

The genesis of this note was a supposedly straightforward exercise, based on a simple initial-boundary value problem for linearly dispersive waves in a periodic domain, that I had devised for my forthcoming text in partial differential equations, [10]. Constructing the Fourier series solution is not especially challenging, and so I also asked for graphs of the solution at various times. In the course of writing up the solution, the initial plots that I produced with MATHEMATICA were more or less as predicted; however, when I introduced a different time step, the solution exhibited a completely unexpected behavior. Further experimentation revealed that the solution has a fractal-like, non-differentiable structure at irrational times, but is piecewise constant at rational times! I had never seen anything like this before, but the fact that the problem was so elementary convinced me that it must be well known. Nevertheless, all of the leading experts in dispersive waves to whom I showed these computations were similarly surprised, convincing me that

$\dagger$ Supported in part by NSF Grant DMS 11-08894.

May 1, 2020 
I might be onto something new and of potential significance. Some further digging revealed that such rational/irrational behavior had already been noted in the context of the linear Schrödinger equation, and leads rapidly into the deep waters of advanced Fourier analysis and exponential sums in number theory. This in turn pointed me towards a recent series of papers by Michael Berry and his collaborators, $[\mathbf{1}, \mathbf{3}, \mathbf{4}]$, that describe the Talbot effect in optics and in quantum revivals, which was named after a striking optical experiment, [15], conducted in 1836 by William Henry Fox Talbot, one of the founders of photography. Thus, the present note reveals that a similar Talbot-like quantized/fractal effect can also be found in a broad range of dispersive media.

Although this paper only makes a modest mathematical contribution, the fact that these phenomena are not well known has convinced me that it is worth setting down in print. The paper contains more questions than results, and the implications for wave mechanics, both linear and nonlinear, and numerics remain to be explored.

\section{Dispersion in a Periodic Domain.}

The starting point is the following periodic initial-boundary value problem on the interval $0 \leq x \leq 2 \pi$ for the most basic linearly dispersive wave equation:

$$
\begin{gathered}
\frac{\partial u}{\partial t}=\frac{\partial^{3} u}{\partial x^{3}}, \quad u(0, x)=f(x), \\
u(t, 0)=u(t, 2 \pi), \quad \frac{\partial u}{\partial x}(t, 0)=\frac{\partial u}{\partial x}(t, 2 \pi), \quad \frac{\partial^{2} u}{\partial x^{2}}(t, 0)=\frac{\partial^{2} u}{\partial x^{2}}(t, 2 \pi) .
\end{gathered}
$$

The underlying partial differential equation is called dispersive because, as we shall see, periodic waves of different frequencies move with different speeds. We take as initial data the unit step function:

$$
f(x)=\sigma(x)= \begin{cases}0, & 0<x<\pi, \\ 1, & \pi<x<2 \pi .\end{cases}
$$

The precise values assigned at its discontinuities are not important, although choosing

$f(x)=\frac{1}{2}$ at $x=0, \pi, 2 \pi$ is consistent with Fourier analysis. The boundary conditions allow us to extend the initial data and solution to be $2 \pi$ periodic functions in $x$.

To construct the solution, we begin by expressing it as a time-dependent (complex) Fourier series

$$
u(t, x) \sim \sum_{k=-\infty}^{\infty} b_{k}(t) e^{\mathrm{i} k x},
$$

where, in conformity with the conventions of Fourier analysis, we use $\sim$ rather than $=$ to indicate that the Fourier series is formal and, without additional assumptions or analysis, its convergence is not guaranteed. Substituting (3) into the partial differential equation in (1), and then equating the coefficients of the individual exponentials, we find that each $b_{k}(t)$ satisfies an elementary linear ordinary differential equation:

$$
\frac{d b_{k}}{d t}=-\mathrm{i} k^{3} b_{k}(t), \quad \text { and hence } \quad b_{k}(t)=b_{k}(0) e^{-\mathrm{i} k^{3} t} .
$$


We conclude that any $2 \pi$ periodic solution to the dispersive wave equation has Fourier expansion

$$
u(t, x) \sim \sum_{k=-\infty}^{\infty} b_{k}(0) e^{\mathrm{i}\left(k x-k^{3} t\right)} .
$$

Observe that the solution (4) is $2 \pi$ periodic in both $t$ and $x$. Moreover, since the $k$-th summand is a function of $x-k^{2} t$, periodic waves of frequency $k$ move with wave speed $k^{2}$, thereby justifying the dispersive nature of the system.

To solve the initial value problem, we expand the initial condition in a Fourier series:

$$
f(x) \sim \sum_{k=-\infty}^{\infty} c_{k} e^{\mathrm{i} k x}, \quad \text { where } \quad c_{k}=\frac{1}{2 \pi} \int_{0}^{2 \pi} f(x) e^{-\mathrm{i} k x} d x .
$$

Equating $u(0, x)=f(x)$, we conclude that $b_{k}(0)=c_{k}$ are the Fourier coefficients of the initial data. In the case of the step function (2), the Fourier coefficients are

$$
b_{k}(0)= \begin{cases}\mathrm{i} /(\pi k), & k \text { odd }, \\ 1 / 2, & k=0, \\ 0, & 0 \neq k \text { even. }\end{cases}
$$

Inserting these particular values into the general solution formula (4), and rewriting the result in terms of real trigonometric functions, we obtain the (formal) Fourier expansion of the solution to the original initial-boundary value problem (1-2):

$$
u^{\star}(t, x) \sim \frac{1}{2}-\frac{2}{\pi} \sum_{j=0}^{\infty} \frac{\sin \left((2 j+1) x-(2 j+1)^{3} t\right)}{2 j+1} .
$$

Let us now graph the solution. We will do this naïvely, using MATHEMATiCA to sum the first 1000 terms in the Fourier series (7). The results, at several times uniformly spaced by $\Delta t=.1$, are plotted in the following pictures:

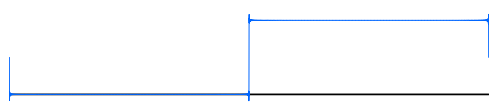

$t=0$.

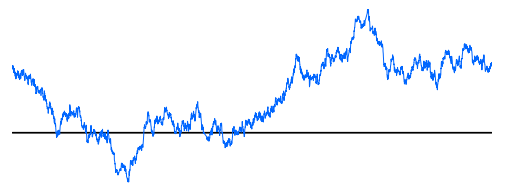

$t=.3$

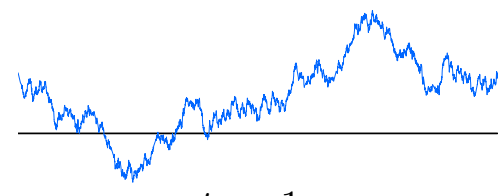

$t=.1$

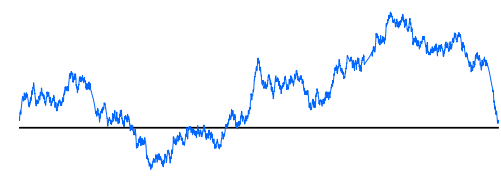

$t=.4$
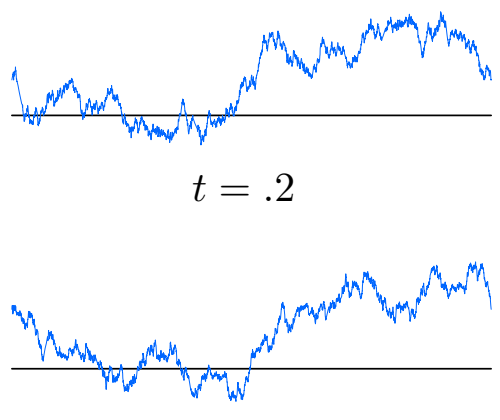

$t=.5$

In the initial graph, you can see a tiny residual Gibbs phenomenon due to the Fourier series approximation. The solution evidentally takes on a fractal-like profile, reminiscent of Weierstrass' continuous but nowhere differentiable function, [7; pp. 401-421]. The temporal evolution continues in this fashion, seemingly at random, until reforming the initial 
data at $t=2 \pi$, after which the process periodically repeats. Incidentally, plotting the solution at a much smaller time step makes the dispersive effects of the initial discontinuity fully manifest:

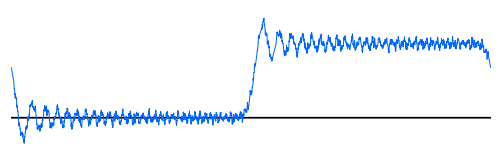

$t=.0001$

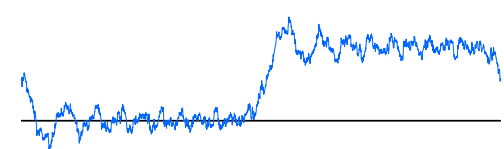

$t=.001$

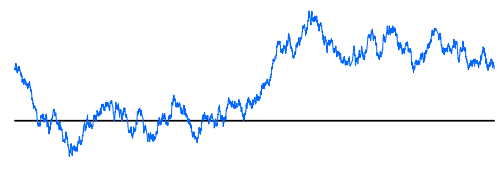

$t=.01$

So, while the fractal nature of the solution is mildly intriguing, most people with some familiarity with dispersive wave phenomena would probably not be unduly surprised by the observed behavior. However, let us instead graph the solution (7) at times spaced by $\Delta t=\frac{1}{30} \pi \approx .10472$. Now the results look startingly different:

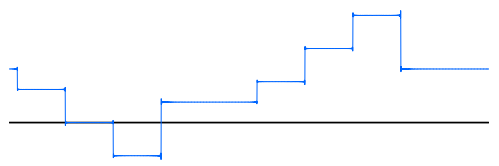

$t=\frac{1}{30} \pi$

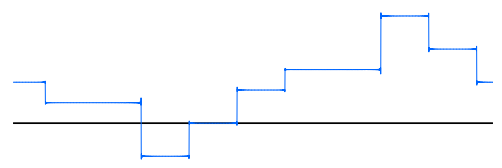

$t=\frac{2}{15} \pi$

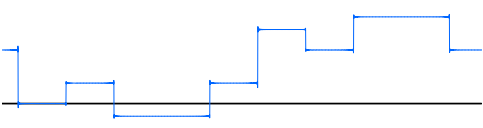

$t=\frac{1}{15} \pi$

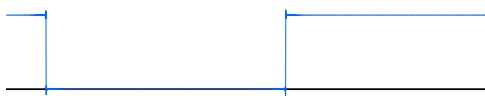

$t=\frac{1}{6} \pi$

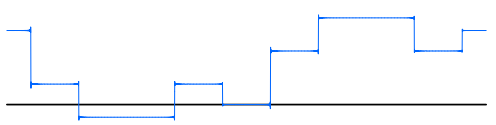

$t=\frac{1}{10} \pi$

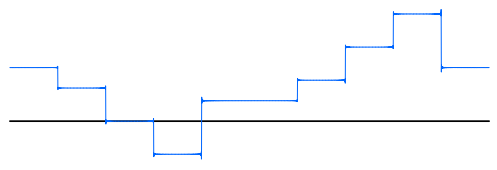

$t=\frac{1}{5} \pi$

Apparently, at these times, the solution is piecewise constant! Here are some additional solution graphs at various rational multiples of $\pi$ :

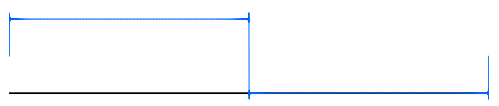

$t=\pi$

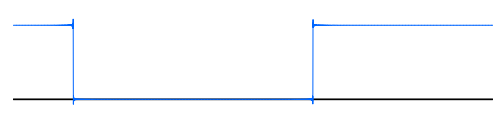

$t=\frac{1}{4} \pi$

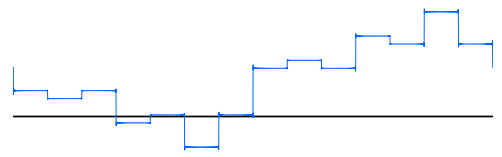

$t=\frac{1}{7} \pi$

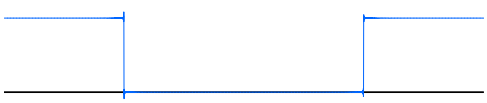

$t=\frac{1}{2} \pi$

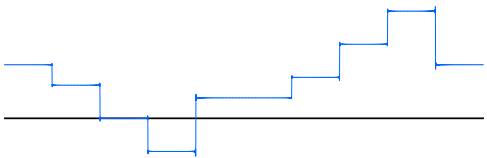

$t=\frac{1}{5} \pi$

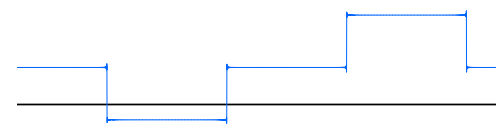

$t=\frac{1}{8} \pi$

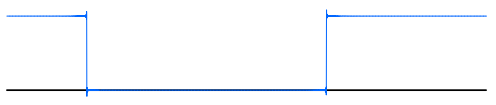

$t=\frac{1}{3} \pi$

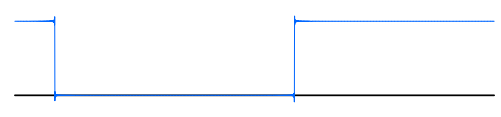

$t=\frac{1}{6} \pi$

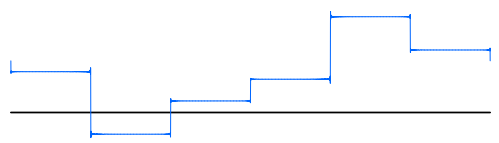

$t=\frac{1}{9} \pi$

While the detailed structure of these solution profiles is not completely clear, it is becoming evident that, at rational multiples of $\pi$, the solution to the initial value problem 
(1) is piecewise constant. Of course, one might be tempted to ascribe this to some strange numerical artifact, but further computational experiments make this extremely unlikely. And, indeed, there is a relatively easy proof of the relevant result.

Theorem 1. Let $p / q \in \mathbb{Q}$ be a rational number ${ }^{\dagger}$. Then the solution (7) to the initial-boundary value problem (1) at time $t=\pi p / q$ is constant on every subinterval $\pi j / q<x<\pi(j+1) / q$ for $j=0, \ldots, 2 q-1$.

Proof: The first step is to note that any function $h(x)$ that satisfies the conclusions of the theorem is a linear combination,

$$
h(x)=\sum_{j=0}^{2 q-1} a_{j} \sigma^{j, q}(x),
$$

of the compressed box functions

$$
\sigma^{j, q}(x)=\left\{\begin{array}{ll}
1, & \pi j / q<x<\pi(j+1) / q, \\
0, & \text { otherwise, }
\end{array} \quad 0 \leq j \leq 2 q-1 .\right.
$$

The box function (9) has Fourier coefficients

$$
\begin{aligned}
c_{k}^{j, q}=\frac{1}{2 \pi} \int_{0}^{2 \pi} \sigma^{j, q}(x) e^{-\mathrm{i} k x} d x & =\frac{1}{2 \pi} \int_{\pi j / q}^{\pi(j+1) / q} e^{-\mathrm{i} k x} d x \\
& = \begin{cases}\frac{\mathrm{i}\left(e^{-\mathrm{i} \pi k / q}-1\right)}{2 \pi k} e^{-\mathrm{i} \pi j k / q}, & k \neq 0, \\
\frac{1}{2 q}, & k=0 .\end{cases}
\end{aligned}
$$

Thus, the Fourier coefficients of the piecewise constant function (8) are

$$
c_{k}=\sum_{j=0}^{2 q-1} a_{j} c_{k}^{j, q} .
$$

As a consequence, Theorem 1 will be established if we can show that the Fourier coefficients $b_{k}(t)$ of our solution (7) at time $t=\pi p / q$ have the form (11) for suitable $a_{0}, \ldots, a_{2 q-1}$. Let us analyze what this entails.

Observe that, given $c_{-q}, \ldots, c_{q-1}$, the equations in (11) corresponding to indices $k=$ $-q,-q+1, \ldots, q-1$ form a system of $2 q$ linear equations for the $2 q$ unknowns $a_{0}, \ldots, a_{2 q-1}$. Moreover, by (10), the rescaled Fourier coefficients

$$
\widehat{c}_{k}= \begin{cases}c_{0}, & k=0, \\ c_{k}=0, & 0 \neq k \equiv 0 \bmod 2 q, \\ \frac{\pi k}{\mathrm{i} q\left(e^{-\mathrm{i} \pi k / q}-1\right)} c_{k}, & k \neq 0 \bmod 2 q,\end{cases}
$$

$\dagger$ Throughout, we assume that the fraction $p / q$ is in lowest terms, i.e., $p$ and $q$ have no common factors. 
satisfy

$$
\widehat{c}_{k}=\frac{1}{2 q} \sum_{j=0}^{2 q-1} a_{j} e^{-\mathrm{i} \pi j k / q} .
$$

Thus, the $2 q$ rescaled coefficients $\widehat{c}_{-q}, \ldots, \widehat{c}_{q-1}$ coincide with the discrete Fourier transform (DFT), [11], of the data $a_{0}, \ldots, a_{2 q-1}$ specified by the piecewise constant function (8). We immediately conclude that the linear system (11) has a unique solution, which is provided by the DFT reconstruction formula

$$
a_{j}=\sum_{l=-q}^{q-1} \widehat{c}_{l} e^{\mathrm{i} \pi l j k / q}, \quad j=0, \ldots, 2 q-1 .
$$

Finally, again thanks to (10), the rescaled Fourier coefficients (12) satisfy

$$
\widehat{c}_{k}=\widehat{c}_{l}, \quad k \equiv l \not \equiv 0 \bmod 2 q, \quad \widehat{c}_{k}=0, \quad 0 \neq k \equiv 0 \bmod 2 q .
$$

Thus, a Fourier series corresponds to a function that is constant on every subinterval $\pi j / q<x<\pi(j+1) / q$ if and only if its Fourier coefficients satisfy (15). This connection between the discrete Fourier transform and Fourier series of piecewise constant functions is, of course, not new.

Now, the Fourier coefficients of our solution (7) at the rational time $t=\pi p / q$ are

$$
c_{k}=b_{k}\left(\pi \frac{p}{q}\right)=b_{k}(0) e^{-\mathrm{i} k^{3} \pi p / q}
$$

where $b_{k}(0)$ are given by (6). The crucial observation is that

$$
\text { if } k \equiv l \bmod 2 q, \text { then } k^{3} \equiv l^{3} \bmod 2 q, \text { and so } e^{-\mathrm{i} k^{3} \pi p / q}=e^{-\mathrm{i} l^{3} \pi p / q} \text {. }
$$

This implies that the corresponding rescaled Fourier coefficients (12) satisfy the piecewise constant criteria (15), which justifies the statement of the Theorem.

Q.E.D.

Remark: Although we have focussed on the step function, the argument immediately generalizes to any initial data $u(0, x)=f(x)$ that is piecewise constant on the same subintervals of length $\pi / q$.

This explains most of the features of the preceding quantized solution graphs. Sometimes, the solution assumes identical values on adjacent subintervals, and so exhibits larger regions of constancy. There is no doubt some number-theoretic characterization of the lengths of such subintervals, but so far I haven't spotted the pattern.

So what's going on? At irrational times (relative to $\pi$ ) the solution has a nondifferentiable, fractal-like nature. On the other hand, at rational times, the solution is piecewise constant — the dispersion has, curiously, "quantized" the solution. These quantized solution profiles are densely embedded in the fractal regime, so that the fractalized, irrational profiles are limits of quantized, rational profiles at progressively finer and finer scales. And thus, somehow the dispersive evolution is able to distinguish rational from irrational times. 
At this point, one might raise the objection that this particular solution is not continuous, and so its status as a weak solution ${ }^{\dagger}$ to the dispersive wave equation is not entirely clear. Of course, one can mollify the solution by convolution with a smooth, concentrated kernel, which would effectively smooth out the coarser quantized times.

Since the underlying partial differential equation is linear, with constant coefficients, derivatives and integrals of solutions are also solutions. In particular, integrating the solution (7) with respect to $x$ a couple of times (omitting the constant terms in order to preserve periodicity) produces a bona fide classical solution whose derivatives exhibit such quantization/fractalization behavior. Vice versa, differentiating the solution (7) once with respect to $x$ yields a linear combination of two fundamental solutions, which corresponds to a pair of initial unit delta function impulses, of opposite strength, concentrated at positions $x=0$ and $x=\pi$.

Specifically, the fundamental solution $u=F(t, x)$ based at the point $x=\pi$, say, corresponds to the initial data $u(0, x)=\delta(x-\pi)$, where $\delta(x)$ denotes the Dirac delta function (or, more accurately, distribution), $[\mathbf{9}, \mathbf{1 0}]$. Since the periodic boundary value problem is translation-invariant, the fundamental solution resulting from a unit delta impulse at another location $x=\xi$ is simply $u=F(t, x-\xi+\pi)$. Applying the preceding Fourier analysis (3-5), we find that the fundamental solution has the formal Fourier expansion

$$
F(t, x) \sim \frac{1}{2 \pi} \sum_{k=-\infty}^{\infty}(-1)^{k} e^{\mathrm{i}\left(k x-k^{3} t\right)} .
$$

The solution to the general initial value problem (1) is then provided by the superposition formula

$$
u(t, x)=\int_{0}^{2 \pi} F(t, x-\xi) f(\xi) d \xi .
$$

Just as the step function solution is piecewise constant at rational times, the fundamental solution is a superposition of finitely many delta functions when $t$ is a rational multiple of $\pi$. On the other hand, when $t$ is an irrational multiple of $\pi$, the convergence of the fundamental solution's Fourier series (18) is more problematic, being the "derivative of a non-differentiable function". To establish the former result, we invoke the following lemma, whose proof follows a line of reasoning similar to that of Theorem 1; details are left as an exercise.

Lemma 2. The Fourier coefficients (5) of a function $f(x)$ are $q$-periodic in their indices, so $c_{k+q}=c_{k}$ for all $k$, if and only if the series represents a linear combination of $q$

$\dagger$ By definition, a $2 \pi$ periodic (in both $t$ and $x$ ) function $u(t, x)$ is a weak solution to the dispersive wave equation (1) if and only if $\int_{0}^{2 \pi} \int_{0}^{2 \pi} u(t, x)\left[\varphi_{t}(t, x)-\varphi_{x x x}(t, x)\right] d x d t=0$ for every $2 \pi$ periodic smooth $\left(\mathrm{C}^{\infty}\right)$ function $\varphi(t, x)$. A sufficiently smooth weak solution is a solution in the classical sense - this can be deduced through integrating by parts. Weak solutions are of great importance in the modern theory of partial differential equations, $[\mathbf{9}, \mathbf{1 8}]$, particularly when dealing with solutions having discontinuities, e.g., shock waves. We leave it to the interested reader to explore to what extent our solutions satisfy the weak solution criterion. 
(periodically extended) delta functions concentrated at the rational nodes $x_{j}=2 \pi j / q$ for $j=0,1, \ldots, q-1$ :

for suitable $a_{0}, \ldots, a_{q-1}$.

$$
f(x)=\sum_{j=0}^{q-1} a_{j} \delta(x-2 \pi j / q)
$$

Combining Lemma 2 with (17), we deduce the aforementioned result.

Theorem 3. Suppose $t=\pi p / q$ is a rational multiple of $\pi$. Then the fundamental solution to the initial-boundary value problem (1) is a linear combination of periodically extended delta functions. When $p$ is odd, the $2 q$ delta functions are concentrated at the rational nodes $x_{j}=\pi j / q$ for $j=0, \ldots, 2 q-1$, whereas for $p$ even, the $q$ delta functions are concentrated at $x_{j}=2 \pi j / q$ for $j=0, \ldots, q-1$.

Observe that Theorem 1 can alternatively be deduced as a direct corollary of Theorem 3. Furthermore, applying Theorem 3 to the superposition formula (19) produces the following intriguing corollary:

Corollary 4. At a rational time, any solution profile $u(\pi p / q, x)$ to the periodic initial-boundary value problem depends on only finitely many values of the initial data, namely $u\left(0, x+x_{j}\right)=f\left(x+x_{j}\right)$ where $x_{j}=\pi j / q$ for $j=0, \ldots, 2 q-1$ when $p$ is odd, or $x_{j}=2 \pi j / q$ for $j=0, \ldots, q-1$ when $p$ is even.

Finally, integrating the fundamental solution (18) — leaving aside its constant term to maintain periodicity — results in the Fourier series

$$
G(t, x)=\int_{0}^{x}\left(F(t, y)-\frac{1}{2 \pi}\right) d y \sim \frac{1}{2 \pi \mathrm{i}} \sum_{0 \neq k=-\infty}^{\infty} \frac{(-1)^{k}}{k} e^{\mathrm{i}\left(k x-k^{3} t\right)},
$$

which solves the periodic initial-boundary value problem with piecewise affine initial data ${ }^{\dagger}$

$$
G(0, x)=\int_{0}^{x}\left(F(0, y)-\frac{1}{2 \pi}\right) d y=\int_{0}^{x}\left(\delta(y-\pi)-\frac{1}{2 \pi}\right) d y=\sigma(x)-\frac{x}{2 \pi} .
$$

Thus, when $t=\pi p / q$ is a rational multiple of $\pi$, the integrated fundamental solution is piecewise affine, with overall slope $-1 /(2 \pi)$, while when $t$ is an irrational multiple of $\pi$, it is a fractalized, non-differentiable function. An intriguing observation is that, at rational times $t=\pi p / q$, Fourier series of the form (20) play an important role in number theory [17], where they are known as Weyl exponential sums.

\section{Dispersion, the Schrödinger Equation, and the Talbot Effect.}

The preceding observations can all be straightforwardly extended well beyond the prototypical dispersive wave equation $u_{t}=u_{x x x}$. Recall, [18], that the dispersion relation $\omega(k)$ of a linear, scalar, constant coefficient partial differential equation in one space

$\dagger$ As always, the right hand side really means the $2 \pi$ periodic extension of the indicated function defined on the interval $0<x \leq 2 \pi$. 
dimension is obtained by substituting the exponential solution ansatz

$$
u(t, x)=e^{\mathrm{i}(k x-\omega t)},
$$

and then solving the resulting algebraic equation for the frequency $\omega$ as a function of the wave number $k$. The differential equation is dispersive if the resulting function $\omega(k)$ is real. For example, the third order linearly dispersive wave equation (1) has cubic dispersion relation $\omega(k)=k^{3}$. In general, if $L$ is a scalar, constant coefficient differential operator with purely imaginary Fourier transform $\widehat{L}(k)=\mathrm{i} \varphi(k)$, then the dispersion relation for the scalar evolution equation

$$
\frac{\partial u}{\partial t}=L[u]
$$

is $\omega(k)=\mathrm{i} \widehat{L}(k)=-\varphi(k)$. If the evolution equation is real, this requires that $L$ involve only odd order derivatives.

Now, the only place in the proof of Theorem 1 where the specific form of the partial differential equation played a role was in the key observation (17), whose form follows from the dispersion relation $\omega(k)=k^{3}$ of the underlying partial differential equation. Consequently, the argument retains its validity when the dispersion relation is any polynomial with integer coefficients: $\omega(k) \in \mathbb{Z}[k]$. As a result, Theorem 1 remains true for any scalar evolution equation (22) with integral dispersion relation, when subject to the same piecewise constant initial conditions and periodic boundary conditions. Moreover, the conclusions of Theorem 3 and Corollary 4, describing the form of the fundamental solution and the dependence of general solutions on the initial data at rational times, are also valid. By rescaling time, these results can be readily extended to the case when the coefficients of $\omega(k)$ are integer multiples of a common real number.

These arguments are equally applicable to complex differential operators with integral dispersion relations, and hence, in particular, to the elementary Schrödinger equation

$$
\mathrm{i} \frac{\partial u}{\partial t}=\frac{\partial^{2} u}{\partial x^{2}}
$$

whose dispersion relation is $\omega(k)=-k^{2}$. We conclude that periodic solutions to the Schrödinger equation (23) exhibit the same quantization/fractalization phenomena at rational/irrational times (relative to the length of the interval). The conclusions also apply to any of the standard homogeneous boundary value problems: Dirichlet, Neumann, or mixed, since their solutions can be obtained by restricting suitably periodic extensions. This leads to intriguing implications for basic quantum mechanics on bounded domains.

Now it turns out that such phenomena have already been observed in the context of the linear Schrödinger equation (23). As before, let us concentrate on the periodic initial-boundary value problem

$$
u(t, 0)=u(t, 2 \pi), \quad u_{x}(t, 0)=u_{x}(t, 2 \pi) .
$$

As in [12], the Fourier series for the integrated fundamental solution is given by

$$
u=G(t, x)=\frac{1}{2 \pi \mathrm{i}} \sum_{0 \neq k=-\infty}^{\infty} \frac{(-1)^{k}}{k} e^{\mathrm{i}\left(k x+k^{2} t\right)} .
$$


The preceding arguments will demonstrate that, when $t=\pi p / q$ is a rational multiple of $\pi$, the solution $G(\pi p / q, x)$ is piecewise affine on the appropriate subintervals of length $\pi / q$, and hence the fundamental solution is a linear combination of delta functions concentrated at rational nodes $x_{j}=\pi j / q$. On the other hand, when $t$ is an irrational multiple of $\pi$, (25) takes on a fractal nature, spread out over the entire interval. Oskolkov, [12], in fact proves that, at irrational times, the solution is a continuous but nowhere differentiable function, confirming (at least in the Schrödinger context) our earlier contention for the dispersive wave equation. A contemporaneous paper by Kapitanski and Rodnianski, [8], proves that solutions to the periodic Schrödinger equation have better analytic properties, as measured by Gevrey norms, at irrational times, a result that inspired them to speculate whether a quantum particle "knows the time". The quantization phenomenon at rational times was explicitly noted by Michael Taylor, [16], who applied this fact to deduce sharp estimates on the Schrödinger solution operator at such times.

It is worth pointing out that, when $t=\pi p / q$ is rational, the integrated fundamental solution (25) has the form of a Gauss sum, a concept of great importance in number theory, [17]. Oskolkov, [12], goes on to show how certain basic reciprocity relations for Gauss sums follow from the invariance of the Schrödinger operator under spatial inversions. The relevance of such number-theoretic Gauss and Weyl exponential sums to quantum mechanics can be traced back to work of Berry, Goldberg, and Hannay, [2, 6]. The paper [6] offers a physical interpretation of the dispersive quantization/fractalization phenomena induced by Fresnel diffraction by periodic gratings. The intricate fractal images, dubbed curlicues, traced by partial Gauss sums in the complex plane are investigated in [2].

In fact, as Michael Berry and his collaborators subsequently discovered, $[\mathbf{1}, \mathbf{3}, \mathbf{4}]$, the rational/irrational dichotomy has its origins in a fascinating optical experiment performed in 1836 by Talbot, [15]. Talbot looked through a magnifying lens at images produced by white light passing through a diffraction grating. When the lens was placed close to the grating, the image, as expected, was blurred. However, surprisingly, moving the lens farther away led to a sharply focussed pattern, whose complementary colors changed with distance. Talbot's experiment was revisited by Lord Rayleigh, [13], who calculated the Talbot distance to be $d^{2} / \lambda$, where $d$ is the grating's spacing and $\lambda$ is the light's wavelength. In 1996, Berry and Klein, [3], mathematically justified the Talbot effect by writing the wave function due to an evenly spaced diffraction grating as a Gauss sum of the form (25). Moreover, they discovered that, at rational fractions of the Talbot distance, the sharp image reappears in multiple smaller copies, while at irrational multiples it has a fractal, non-differentiable profile. Indeed, rigorous results on the fractal dimension of the solution graph at irrational times can be found in [14].

The full optical image forms a remarkable multi-hued "Talbot carpet"; some examples can be found in [4]. On the next page we display the "dispersive carpets" corresponding to the particular solutions (7) and (25). The horizontal axis is $0 \leq x \leq 2 \pi$, while the vertical axis is a half period $0 \leq t \leq \pi$. The colors (gray scale) represent solution values, with red (light) being positive and blue (dark) negative. In both cases, despite the presence of complicated quantized/fractal behavior, there is a discernible wave that moves across the interval with unit speed. An explanation of the repeating tilted stripes, though, is less apparent. 


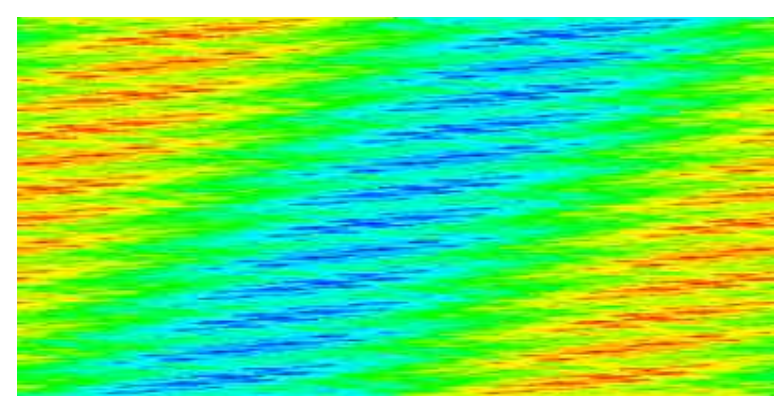

Dispersive Carpet

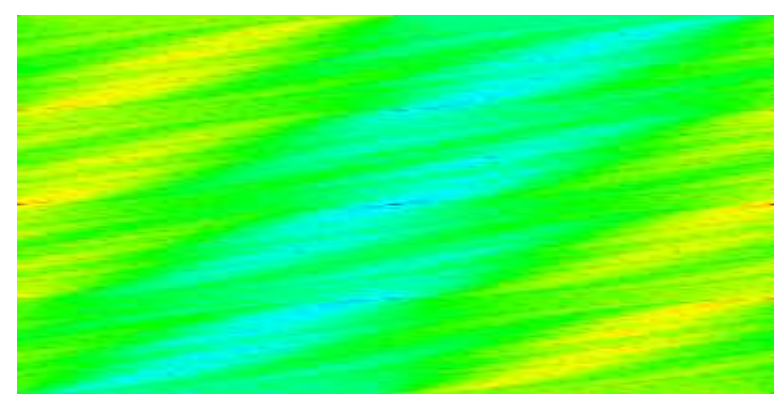

Schrödinger Carpet

Berry and Klein, [3], further noted that the quantized/fractal Talbot effect also appears in the phenomenon of quantum revival, in which an initially concentrated wave function, representing, say, an electron in an atomic orbit, at first spreads out but subsequently relocalizes. Experimental confirmations of the Talbot effect in both optics and atoms are described in [4]. Thus, the observations contained in this note can be viewed as extending the quantized/fractal Talbot effect to a broad range of linearly dispersive wave equations on periodic domains.

\section{Further Directions.}

The elementary but surprising results described here raise many more questions than they answer. Here are a few possible directions for further research.

- We can clearly extend these results to linearly dispersive equations in higher space dimensions. With the appropriate integrality hypothesis on the dispersion relation, the periodic initial-boundary value problem on a rectangle whose initial data is constant on rational subrectangles will result in a solution that is quantized at rational times and fractalized at irrational times. Taylor, [16], establishes similar results for the linear Schrödinger equation on tori, spheres, and even Zoll manifolds. An interesting question is whether similar phenomena arise on more general compact domains. The case of dispersive waves on a sphere, e.g., the earth, is particularly deserving of further investigation.

- It would be interesting to see whether invariance properties of dispersive wave equations lead to novel reciprocity relations and other identities for the associated number-theoretic Weyl exponential sums.

- We have concentrated on the periodic boundary value problem for linearly dispersive wave equations. The behavior under other boundary conditions, e.g., $u(t, 0)=$ $u_{x}(t, 0)=u(t, 2 \pi)=0$, is not so clear because, unlike the Schrödinger equation, these boundary value problems are not naturally embedded in the periodic version. Fokas, [5], has developed a new solution technique for linear partial differential equations based on novel integral representations, and it would be instructive to see how such effects are manifested in his approach.

- How does this complicated analytic behavior impact numerical solution techniques? The fact that different time steps (rational versus irrational) result in radically different solution profiles indicates that the design of numerical solution schemes that 
accurately capture the phenomena will be a challenge. However, practical testing remains to be done. See $[\mathbf{2 0}]$ for a recent survey on the numerics of dispersive partial differential equations.

- Another issue is whether quantization/fractalization impacts dispersive nonlinear partial differential equations on bounded domains. The numerical solution of the periodic Korteweg-deVries equation, first implemented by Zabusky and Kruskal in their seminal discovery of the soliton, [19], appears not to exhibit such behavior. Why doesn't one see such effects in, say, periodic solutions to the Korteweg-deVries or nonlinear Schrödinger equations? Perhaps the nonlinear term suffices to override the anomalous effects of the linear dispersion. Or is the behavior there, and we just haven't looked carefully enough?

- The Talbot effect has been experimentally observed in both optics and atoms, [4]. Can one design experiments that exhibit such behavior in other dispersive media?

Acknowledgments: I'd like to thank Mark Keel, Philip Rosenau, Jerry Bona, Carlos Kenig, Thanasis Fokas, Walter Strauss, Peter Lax, David Levermore, and, particularly, Michael Taylor and Michael Berry, as well as the anonymous referees, for enlightening correspondence, references, and valuable suggestions.

\section{References}

[1] Berry, M.V., and Bodenschatz, E., Caustics, multiply-reconstructed by Talbot interference, J. Mod. Optics 46 (1999), 349-365.

[2] Berry, M.V., and Goldberg, J., Renormalization of curlicues, Nonlinearity 1 (1988), $1-26$.

[3] Berry, M.V., and Klein, S., Integer, fractional and fractal Talbot effects, J. Mod. Optics 43 (1996), 2139-2164.

[4] Berry, M.V., Marzoli, I., and Schleich, W., Quantum carpets, carpets of light, Physics World 14(6) (2001), 39-44.

[5] Fokas, A.S., A Unified Approach to Boundary Value Problems, CBMS-NSF Conference Series in Applied Math., vol. 78, SIAM, Philadelphia, 2008.

[6] Hannay, J.H., and Berry, M.V., Quantization of linear maps on a torus - Fresnel diffraction by a periodic grating, Physica 1D (1980), 267-290.

[7] Hobson, E.W., The Theory of Functions of a Real Variable and the Theory of Fourier's Series, Dover Publ., New York, 1957.

[8] Kapitanski, L., and Rodnianski, I., Does a quantum particle know the time?, in: Emerging Applications of Number Theory, D. Hejhal, J. Friedman, M.C. Gutzwiller and A.M. Odlyzko, eds., IMA Volumes in Mathematics and its Applications, vol. 109, Springer Verlag, New York, 1999, pp. 355-371.

[9] McOwen, R.C., Partial Differential Equations: Methods and Applications, Prentice-Hall, Inc., Upper Saddle River, N.J., 2002. 
[10] Olver, P.J., Introduction to Partial Differential Equations, in preparation. See http://www . math. umn. edu/ olver/pdn.html.

[11] Olver, P.J., and Shakiban, C., Applied Linear Algebra, Prentice-Hall, Inc., Upper Saddle River, N.J., 2006.

[12] Oskolkov, K.I., Schrödinger equation and oscillatory Hilbert transforms of second degree, J. Fourier Anal. Appl. 4 (1998), 341-356.

[13] Rayleigh, L., On copying diffraction-gratings, and on some phenomena connected therewith, Philos. Mag. 11 (1881), 196-205.

[14] Rodnianski, I., Fractal solutions of the Schrödinger equation, Contemp. Math. 255 (2000), 181-187.

[15] Talbot, H.F., Facts related to optical science. No. IV, Philos. Mag. 9 (1836), 401-407.

[16] Taylor, M., The Schrödinger equation on spheres, Pacific J. Math. 209 (2003), $145-155$.

[17] Vinogradov, I.M., The Method of Trigonometrical Sums in the Theory of Numbers, Dover Publ., Mineola, NY, 2004.

[18] Whitham, G.B., Linear and Nonlinear Waves, John Wiley \& Sons, New York, 1974.

[19] Zabusky, N.J., and Kruskal, M.D., Interaction of "solitons" in a collisionless plasma and the recurrence of initial states, Phys. Rev. Lett. 15 (1965), 240-243.

[20] Zuazua, E., Propagation, observation, and control of waves approximated by finite difference methods, SIAM Review 47 (2005), 197-243. 